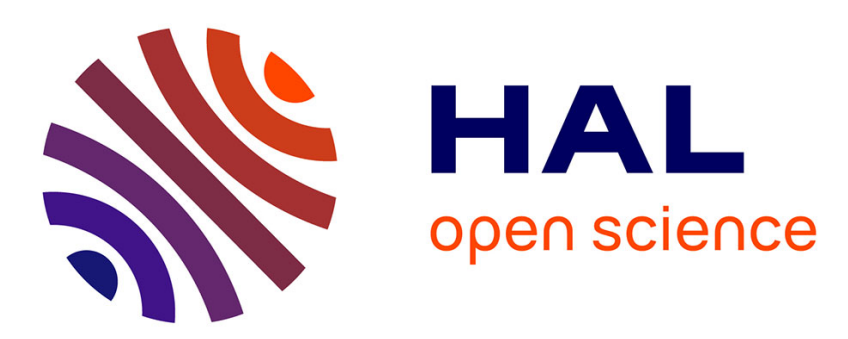

\title{
Rôle des effets non newtoniens dans l'écoulement pulsé d'un fluide dans un tuyau viscoélastique P. Flaud, D. Quemada
}

\section{To cite this version:}

P. Flaud, D. Quemada. Rôle des effets non newtoniens dans l'écoulement pulsé d'un fluide dans un tuyau viscoélastique. Revue de Physique Appliquée, 1980, 15 (3), pp.749-759. 10.1051/rphysap:01980001503074900 . jpa-00244785

\section{HAL Id: jpa-00244785 https://hal.science/jpa-00244785}

Submitted on 1 Jan 1980

HAL is a multi-disciplinary open access archive for the deposit and dissemination of scientific research documents, whether they are published or not. The documents may come from teaching and research institutions in France or abroad, or from public or private research centers.
L'archive ouverte pluridisciplinaire HAL, est destinée au dépôt et à la diffusion de documents scientifiques de niveau recherche, publiés ou non, émanant des établissements d'enseignement et de recherche français ou étrangers, des laboratoires publics ou privés. 


\title{
Rôle des effets non newtoniens dans l'écoulement pulsé d'un fluide dans un tuyau viscoélastique
}

\author{
P. Flaud et D. Quemada \\ Laboratoire de Biorhéologie et d'Hyd rodynamique Physiologique. \\ Equipe de Recherche $\left({ }^{*}\right)$, 2, place Jussieu, 75221 Paris Cedex 05, France
}

(Reçu le 7 août 1979, révisé le 3 décembre 1979, accepté le 6 décembre 1979)

\begin{abstract}
Résumé. - Les effets non newtoniens dans les écoulements de dispersions concentrées, dans des conduites viscoélastiques de grandes dimensions, devant celles des particules, sont étudiés. Différentes lois de comportement pseudo-plastique sont utilisées, et le caractère pulsé de l'écoulement est pris en compte. Des variations notables des profils de vitesse (aplatissement), et des débits moyens (diminution) sont mises en évidence, qui dépendent des caractéristiques géométriques et rhéologiques du fluide et de la paroi du conduit. L'application aux écoulements sanguins est présentée.
\end{abstract}

\begin{abstract}
Effects of non newtonian behaviour of concentrated dispersions on flow in viscoelastic tubes are studied, assuming tube to particle diameter ratio very large. Different shear-thinning relations have been used, and pulsatile flow has been considered. Significant variations in velocity profiles (blunting) and in mean flow rate (decreasing) have been obtained, which depend on geometrical and rheological characteristics of fluid and tube wall. Application to blood flow has been presented.
\end{abstract}

1. Introduction. - L'importance de la prise en compte des propriétés rhéologiques pariétales dans l'étude des écoulements pulsatiles a été montrée dans un grand nombre de travaux $([1,2,3])$ et notamment lorsque la paroi possédait des propriétés non linéaires. Toutes ces études supposaient des propriétés newtoniennes pour le fluide s'écoulant dans le tuyau. Néanmoins, lorsqu'il s'agit d'un milieu dispersé, il est important de s'assurer de la validité de cette approximation, et, lorsque le fluide ne peut être considéré comme newtonien, de déterminer l'influence des propriétés du fluide sur l'écoulement.

Le travail qui est présenté ici concerne les écoulements développés, stationnaires ou instationnaires d'un fluide non newtonien en tuyau souple. A titre d'illustration, c'est l'écoulement du sang dans le système vasculaire qui sera étudié. Bien qu'il soit communément admis que, dans les gros vaisseaux, le sang peut être assimilé à un fluide newtonien, on verra qu'il convient d'apporter des limitations à une telle assertion.

En effet, cette hypothèse ne prend en compte que

(*) Associée au C.N.R.S. les variations de la viscosité en fonction de la concentration en volume $C$, mais cette concentration est supposée constante en tout point de la section, c'està-dire indépendante de la vitesse de cisaillement. Rappelons que l'on ne peut définir cette viscosité que si le milieu dispersé peut être considéré comme quasi homogène, c'est-à-dire si la dimension $2 a$ des particules est assez petite devant le diamètre moyen $2 R$ du tuyau $\left(a / R \lesssim 10^{-3}\right)$. Pour le sang (suspension essentiellement constituée de globules rouges de diamètre $2 a \sim 10 \mu$ ) s'écoulant dans des gros vaisseaux $(1 \mathrm{~mm}<2 R<2 \mathrm{~cm})$, cette approximation quasi homogène est donc satisfaite.

Cette approximation newtonienne pour le fluide est habituellement justifiée par l'analyse de la viscosité apparente $\mu_{\mathrm{a}}$ (qui est la viscosité d'un fluide newtonien qui sous l'action du même gradient de pression $\Delta p / L$ s'écoulerait avec le même débit $\left.Q, \mu_{\mathrm{a}}=\pi R^{4} \Delta p /(8 Q L)\right)$ en fonction du cisaillement apparent $\dot{\gamma}_{\mathrm{a}}$ (défini à partir du débit $\dot{\gamma}_{\mathrm{a}}=4 Q /\left(\pi R^{3}\right)$ ) qui correspond donc au gradient de vitesse à la paroi de l'écoulement de Poiseuille correspondant. Ecrite avec la contrainte à la paroi $\tau_{\mathrm{a}}=\Delta P . R /(2 L)$, la relation pression débit peut évidemment s'écrire $\tau_{\mathrm{a}}=\mu_{\mathrm{a}} \dot{\gamma}_{\mathrm{a}}$ dont les variations se représentent (Fig. 1) par une courbe confondue avec une droite passant par l'origine, dans la région des 


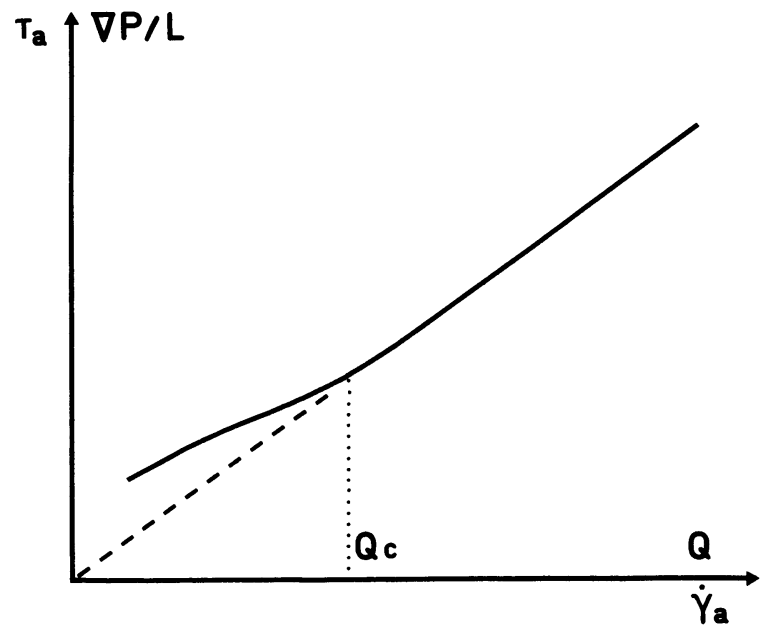

Fig. 1. - Allure de la variation de la contrainte de cisaillement à la paroi $\tau_{\mathrm{a}}$ en fonction de la vitesse de cisaillement $\dot{\gamma}_{\mathrm{a}}$ (écoulement de Couette) ou de la variation du gradient de pression $\Delta P / L$ en fonction du débit $Q:-$ Fluide non newtonien; --.-.- Fluide newtonien.

[Example of variations of wall shear stress $\tau_{\mathrm{a}}$ as a function of shear rate $\dot{\gamma}_{\mathrm{a}}$ (Couette flow), or of pressure gradient $\Delta P / L$ as a function of flow rate $Q:-$ Non newtonian fluid; -.-.-- Newtonian fluid.]

$\dot{\gamma}_{\text {a }}$ (ou $Q$ ) assez grands, mais qui s'incurve vers l'axe des $\tau_{\mathrm{a}}$ pour des $\dot{\gamma}_{\mathrm{a}}$ (ou $Q$ ) très faibles [4]. C'est sans doute l'existence de cette région linéaire, dans le domaine des débits physiologiques, qui a été considérée comme pouvant justifier l'approximation newtonienne.

Ine telle approximation est cependant criticable. En effet, si l'on suppose une viscosité locale non newtonienne $\mu=\mu(\dot{\gamma})$ dès que $\dot{\gamma} \lesssim \dot{\gamma}_{1}$ les effets non newtoniens interviend ront principalement dans une région voisine de l'axe du tuyau (où $\dot{\gamma}=0$ par raison de symétrie), définie par $|\mathrm{d} v / \mathrm{d} r|<\dot{\gamma}_{1}$. De plus, si la concentration $C$ croît (ce qui accentue les effets non newtoniens), le profil de vitesse s'aplatira et la région axiale concernée sera plus étendue tandis que le cisaillement à la paroi, supérieur à $\dot{\gamma}_{\mathrm{a}}$, augmentera. De tels profils de vitesse ont été très souvent observés. Le domaine de validité de l'approximation newtonienne ne peut donc pas être considéré comme clairement établi.

Le présent travail a pour but la discussion de cette validité à travers l'évaluation des effets non newtoniens sur l'écoulement dans les gros vaisseaux sanguins, mais il pourra être appliqué à de nombreux écoulements de fluides non newtoniens dans des conduites larges.

Avant d'aborder cette discussion, il est nécessaire de présenter les relations de comportement qui permettront de décrire de manière satisfaisante les propriétés rhéologiques du fluide dans la gamme des vitesses de cisaillement rencontrées dans le système.

Dans le cas où l'on considère un écoulement stationnaire, la plus simple de ces relations de comportement permet encore une description satisfaisante de cet écoulement, et c'est cette relation qui sera utilisée par la suite.

Dans le cas où le fluide considéré manifeste un comportement viscoélastique ou thixotrope, on discutera de la manière dont il convient de prendre cet effet en compte.

Il sera ensuite possible d'étudier l'écoulement d'un fluide non newtonien tel que le sang, par exemple, dans un tuyau déformable, viscoélastique, sous l'action d'un gradient de pression pulsatile, et de montrer dans quelles conditions les propriétés rhéologiques particulières du fluide doivent être prises en compte au même titre que celles de la paroi.

2. Choix du modèle rhéologique. - De nombreux modèles rhéologiques ont été proposés pour décrire le comportement non newtonien des milieux dispersés. Parmi ces modèles, certains sont purement phénoménologiques, et d'autres cherchent à relier plus précisément les paramètres intervenant dans le modèle aux différents phénomènes physiques qu'il convient de prendre en compte (concentration, déformabilité, agrégation des particules...). Les modèles qui seront présentés ici relient la contrainte de cisaillement $\tau$ au gradient de vitesse $\dot{\gamma}$ dans un cisaillement simple.

2.1 Modèle de Casson pseudoplastique. - Le comportement rhéologique du sang et des milieux dispersés concentrés est relativement bien représenté par le modèle de Casson [5] défini par la relation :

$$
\begin{aligned}
\tau>\tau_{0} & \tau^{1 / 2}=\tau_{0}^{1 / 2}+k \dot{\gamma}^{1 / 2} \\
\tau<\tau_{0} & \dot{\gamma}=0 .
\end{aligned}
$$

Dans cette relation $k$ est une constante qui peut être reliée à la viscosité apparente du fluide pour des forts cisaillements $\mu_{\infty}$ :

$$
k=\mu_{\alpha}^{1 / 2}
$$

et $\tau_{0}$ une contrainte seuil en dessous de laquelle le fluide se comporte comme un solide.

Il convient de noter qu'au-delà d'une certaine valeur de $\dot{\gamma}$ (cf. [6, 4]), le fluide retrouve un comportement newtonien et que la relation précédente (1) ne se trouve plus vérifiée. Ainsi pour du sang humain dans un état non pathologique cette valeur limite de la vitesse de cisaillement est de l'ordre de $100 \mathrm{~s}^{-1}$.

D'autre part, l'existence de la contrainte seuil est controversée et la discontinuité qu'elle représente semble physiquement difficilement acceptable. Aussi se propose-t-on de remplacer ce modèle à deux paramètres par le modèle à trois paramètres suivant :

$$
\tau=\dot{\gamma}\left(\frac{\tau_{0}^{1 / 2}}{|\dot{\gamma}|^{1 / 2}} \tanh \left(\alpha\left|\frac{\mu_{\alpha} \dot{\gamma}}{\tau_{0}}\right|^{1 / 2}\right)+\mu_{\alpha}^{1 / 2}\right)^{2} .
$$

On peut vérifier que l'on retrouve la relation (1), c'està-dire la loi de Casson lorsque $j \gg \tau_{0} \alpha^{2} / \mu$, ou encore 
quand $\alpha \rightarrow \infty$. On peut déduire de (2) la viscosité apparente à vitesse de cisaillement nulle qui s'écrit :

$$
\mu_{0}=\mu_{\alpha}(1+\alpha)^{2} \text {. }
$$

2.2 Modèle de Bingham Continu. - On étudiera également le modèle suivant qui est identique au modèle de Bingham pour les vitesses de cisaillement importantes et qui est défini par la relation :

$$
\tau=\tau_{\mathrm{b}} \tanh \left(\beta \frac{\mu_{1} \dot{\gamma}}{\tau_{\mathrm{b}}}\right)+\mu_{1} \dot{\gamma}
$$

qui redonne le modèle de Bingham classique quand $\beta \rightarrow \infty$.

Dans ce modèle la viscosité apparente à cisaillement nul s'écrit :

$$
\mu_{0}=\mu_{1}(1+\beta) .
$$

2.3 MODÈLE DE RÉFÉRENCE. - Une théorie a récemment été développée [7] qui se base sur un principe de dissipation minimale de l'énergie. Elle aboutit à la détermination d'un modèle rhéologique où la viscosité est liée à l'état structurel du fluide et que l'on peut écrire, en l'étendant au domaine de vitesse de cisaillement $(-\infty,+\infty)$

$$
\tau=\frac{\mu_{\mathrm{p}} \dot{\gamma}}{\left(1-\frac{C}{2} \frac{k_{0} \dot{\gamma}_{c}^{1 / 2}+k_{\alpha}|\dot{\gamma}|^{1 / 2}}{\dot{\gamma}_{c}^{1 / 2}+|\dot{\gamma}|^{1 / 2}}\right)^{2}} .
$$

Dans ce modèle $\mu_{\mathrm{p}}$ est la viscosité dynamique du milieu suspendant, et $C$ la concentration en volume des particules suspendues; $k_{0}, k_{\alpha}$ et $\dot{\gamma}_{c}$ sont des paramètres caractéristiques qui peuvent être reliés à l'état d'agrégation, de déformation, et à un temps moyen de relaxation de la structure.

$\mathrm{Ce}$ modèle est particulièrement intéressant dans la mesure où il permet une bonne interprétation de résultats expérimentaux dans des conditions très variées. Il servira, ici, de modèle de référence, à fin de comparaison avec les autres modèles proposés.

2.4 COMParaison DES DIFFÉRENTS MODÈles. -

En se plaçant dans le cas particulier où le milieu à étudier est du sang il est possible de comparer les différentes relations proposées. Dans la relation proposée par Quemada [7] on prendra comme valeur des paramètres $\mu_{\mathrm{p}}, k_{\infty}, k_{0}, \dot{\gamma}_{\mathrm{c}}$ et $C$, les valeurs suivantes correspondant aux valeurs obtenues pour du sang humain dans des conditions non pathologiques :

$$
\begin{gathered}
\mu_{\mathrm{p}}=1,2 \times 10^{-3}(\mathrm{MKS}) \quad k_{\infty}=1,78 \quad k_{0}=4,20 \\
\dot{\gamma}_{\mathrm{c}}=5 \mathrm{~s}^{-1} \quad C=0,45 .
\end{gathered}
$$

On a représenté (Fig. 2), dans un diagramme $\tau^{1 / 2}\left(\dot{\gamma}^{1 / 2}\right)$ les différentes relations proposées précédemment. On remarque en particulier qu'il est possible d'ajuster les paramètres intervenant dans le modèle de
Casson continu de manière à obtenir un excellent accord avec le modèle de référence, le modèle de Bingham continu n'étant en bon accord que pour des vitesses de cisaillement supérieures à $5 \mathrm{~s}^{-1}$.

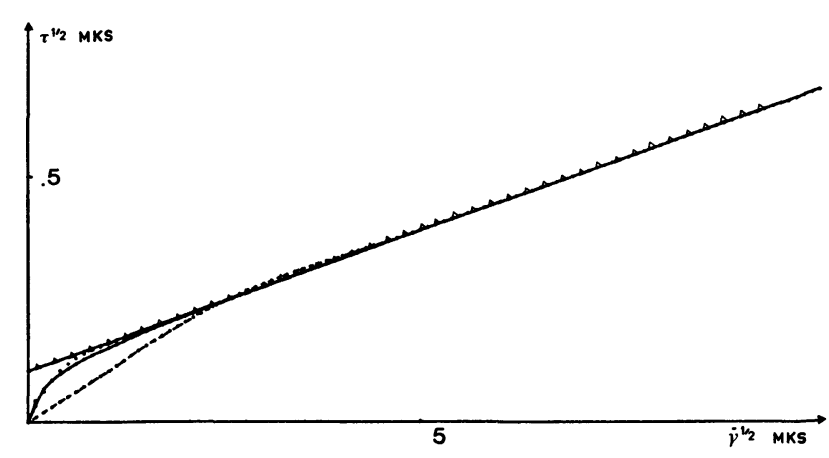

Fig. 2. - Comparaison des différents modèles pour du sang humain non pathologique. Modèle de référence (Quemada) avec $\mu_{\mathrm{p}}=1,2 \times 10^{-3}(\mathrm{MKS}), k_{\infty}=1,7, k_{0}=4,2, \dot{\gamma}_{\mathrm{c}}=5 \mathrm{~s}^{-1}$, $c=0,45 . \Delta \Delta \Delta$ Modèle de Casson (1) avec $\tau_{0}=1,1 \times 10^{-2} \mathrm{~N} . \mathrm{m}^{-2}$, $k=5,7 \times 10^{-2}$ MKS. .... Modèle de Casson continu (IV.2) avec $\tau_{0}=1,1 \times 10^{-2}, \mu_{\infty}=3,2 \times 10^{-3} \mathrm{MKS}, \alpha=5$. ---- Modèle de Bingham continu (IV.3) avec $\tau_{\mathbf{b}}=5 \times 10^{-2}$ N.m ${ }^{-2}$, $\mu_{1}=4,1 \times 10^{-3} \mathrm{MKS}, \beta=1,7$.

[Comparison between different normal human blood models. Shear thinning model (Quemada) $\left(\mu_{\mathrm{p}}=1.2 \times 10^{-3} \mathrm{MKS}\right.$, $\left.k_{\infty}=1.7, k_{0}=4.2, \gamma_{\mathrm{c}}=5 \mathrm{~s}^{-1}, c=0.45\right) . \Delta \Delta \Delta$ Casson model $(1)$ $\left(\tau_{0}=1.1 \times 10^{-2}\right.$ N.m ${ }^{-2}, k=5.7 \times 10^{-2}$ MKS $) . .$. Continuous Casson model $\left(\tau_{0}=1.1 \times 10^{-2} \mathrm{~N} \cdot \mathrm{m}^{-2}, \mu_{\infty}=3.2 \times 10^{-3} \mathrm{MKS}\right.$, $\alpha=5)$. -.-.- Continuous Bingham model $\left(\tau_{\mathrm{b}}=5 \times 10^{-2} \mathrm{~N} \cdot \mathrm{m}^{-2}\right.$, $\left.\mu_{1}=4.1 \times 10^{-3} \mathrm{MKS}, \beta=1.7\right)$.]

On a également cherché à comparer le modèle de référence au modèle de Bingham continu, pour le même sang mais en supposant que celui-ci était totalement désagrégé. Dans ce cas qui correspond à des situations physiologiques que l'on précisera ultérieurement et qui dépendent donc de l'état d'agrégation des particules, le comportement non newtonien du fluide est moins marqué et l'accord entre les deux modèles est tout à fait satisfaisant (Fig. 3).

2.5 EXTENSION DE CES MODÈLES AUX GRANDES vITESSES DE CISAILLEMENT. - On sait qu'au-delà d'une certaine valeur de $\dot{\gamma}$ les relations précédentes ne traduisent plus de manière satisfaisante le comportement de fluides tels que le sang $([6,4])$ : la relation $\tau=\dot{f}(\gamma)$ n'est en effet valable que pour des valeurs de $\dot{\gamma}$ inférieures à une valeur de transition $\dot{\gamma}_{\mathrm{T}}$. Au-delà, le fluide peut être considéré comme newtonien, et l'on peut écrire $\tau=\mu_{\infty} \dot{\gamma}$.

Afin de traduire de manière phénoménologique cette transition, on propose la relation suivante :

où

$$
\tau=\left(f(\dot{\gamma})-\mu_{\infty} \dot{\gamma}\right) g(\dot{\gamma})+\mu_{\infty} \dot{\gamma}
$$

$$
g(\dot{\gamma})=\frac{1}{2}\left(1-\tanh \left(\frac{|\dot{\gamma}|-\dot{\gamma}_{\mathrm{T}}}{\gamma_{\mathrm{LT}}}\right)\right) .
$$




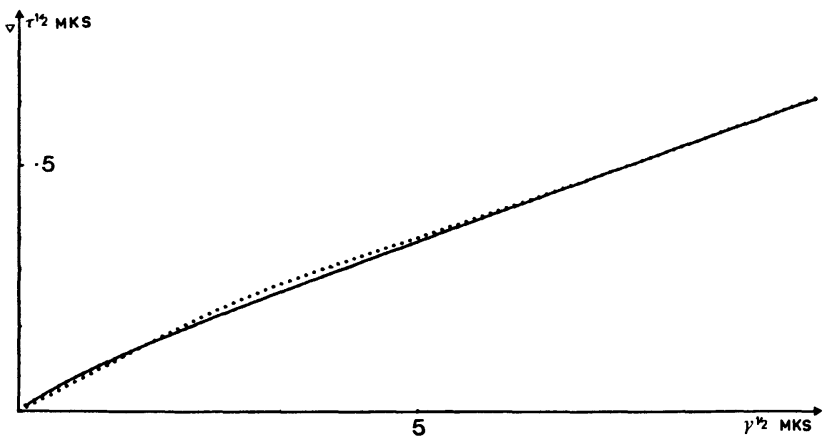

Fig. 3. - Comparaison du modèle de Bingham continu et du modèle de référence pour du sang humain non agrégé. - Modèle de référence avec $\mu_{\mathrm{p}}=1,2 \times 10^{-3} \mathrm{MKS}, k_{\infty}=1,7, k_{0}=3,2$, $\dot{\gamma}_{\mathrm{c}}=7 \mathrm{~s}^{-1}, c=0,45$. -...- Modèle de Bingham continu avec $\tau_{\mathrm{b}}=3,3 \times 10^{-2}, \mu_{1}=3,7 \times 10^{-2} \mathrm{MKS}, \beta=1$.

[Continuous Bingham model in comparison with shear thinning model (non aggregated human blood) : - Shear thinning model $\left(\mu_{\mathrm{p}}=1.2 \times 10^{-3} \mathrm{MKS}, k_{\infty}=1.7, k_{0}=3.2, \dot{\gamma}_{\mathrm{c}}=7 \mathrm{~s}^{-1}, c=0.45\right)$. -- Continuous Bingham model $\left(\tau_{\mathrm{b}}=3.3 \times 10^{-2}\right.$ N. m ${ }^{-2}$, $\left.\mu_{1}=3.7 \times 10^{-3} \mathrm{MKS}, \beta=1\right)$.]

Dans cette relation $\dot{\gamma}_{\mathrm{T}}$ fixe la zone où s'effectue la transition et $\dot{\gamma}_{L T}$ permet d'ajuster la largeur de la zone de transition.

La figure 4 illustre la manière dont une telle relation peut traduire des données expérimentales pour des vitesses de cisaillement élevées. Cette relation sera utilisée par la suite dès lors que le domaine de variation de $\dot{\gamma}$ ne permet plus de négliger cette transition entre le comportement non newtonien et newtonien du fluide.

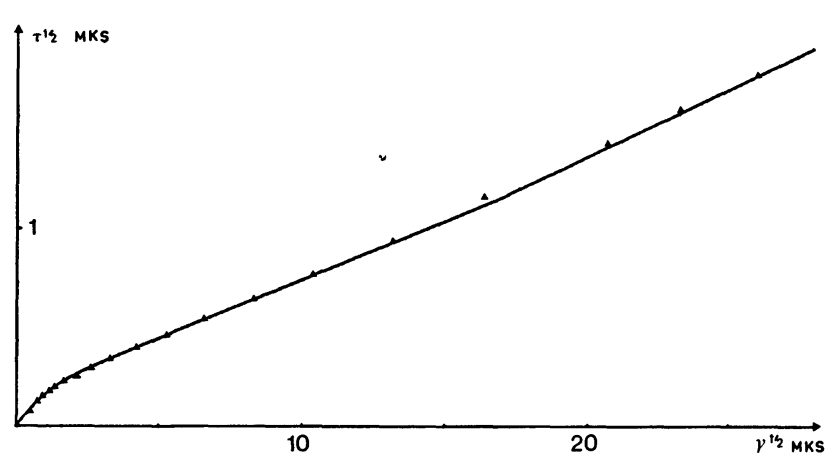

Fig. 4. - Extension des modèles rhéologiques aux grandes vitesses de cisaillement. $\nabla \nabla \nabla$ Données expérimentales obtenues pour du sang humain (hématocrite $=47,6 \%$ ) en présence d'anticoagulant (d'après [6]). — Modèle de Casson continu étendu aux grandes vitesses de cisaillement.

[Generalization of rheological models for high shear rate : $\boldsymbol{\nabla \nabla}$. Experimental data (anticoagulated whole human blood, Hematocrit $=47.6 \%$ ) from [6]. Continuous Casson model extended towards high shear rates.]

3. Profils de vitesse en écoulement stationnaire. 3.1 PrinCIPE DE LA DÉTERMINATION DU PROFIL DE VITESSE. - A partir de la donnée d'un modèle rhéologique décrivant le comportement d'un fluide, il est possible d'étudier l'écoulement laminaire stationnaire et pleinement développé de ce fluide dans un tuyau cylind rique de rayon $R_{0}$ sous l'action d'un gradient de pression donné. Dans une telle géométrie les équations de Navier-Stokes se réduisent à :

$$
\frac{\mathrm{d} p}{\mathrm{~d} z}=\frac{1}{r} \frac{\mathrm{d}}{\mathrm{d} r}(r \tau)
$$

Il est commode d'introduire les variables sans dimension suivantes :

où

$$
\begin{aligned}
& \eta=\frac{r}{R_{0}} \\
& v=\frac{V(r)}{V_{\mathrm{M}}}
\end{aligned}
$$

$$
V_{\mathrm{M}}=-\frac{R_{0}^{2}}{8 \mu_{\infty}} \frac{\mathrm{d} p}{\mathrm{~d} z}
$$

représente la vitesse moyenne d'un écoulement engendré dans le tuyau par le gradient de pression $\mathrm{d} p / \mathrm{d} z$, pour un fluide newtonien de viscosité $\mu_{\alpha}$, viscosité apparente du fluide dans la limite où $\dot{\gamma}$ tend vers l'infini. L'équation (6) se réécrit alors, dans le cas où le fluide est newtonien, ou dans le cas où son comportement rhéologique peut être décrit par une des relations proposées;

- fluide newtonien :

$$
\eta=-\frac{1}{4} \frac{\mathrm{d} v}{\mathrm{~d} \eta}
$$

- modèle de Casson continu :

$$
\begin{aligned}
& \eta=-\frac{1}{4} \frac{\mathrm{d} v}{\mathrm{~d} \eta} \times \\
& \times\left(1+\frac{1}{\left(\delta_{\mathrm{c}}\left|\frac{\mathrm{d} v}{\mathrm{~d} \eta}\right|\right)^{1 / 2}} \tanh \left(\alpha\left|\delta_{\mathrm{c}} \frac{\mathrm{d} v}{\mathrm{~d} \eta}\right|^{1 / 2}\right)\right)^{2}
\end{aligned}
$$

où

$$
\delta_{\mathrm{c}}=\frac{\mu_{\infty} V_{\mathrm{M}}}{\tau_{0} R_{0}}
$$

- modèle de Bingham continu :

$\eta=-\frac{1}{4} \frac{\mathrm{d} v}{\mathrm{~d} \eta} \frac{\mu_{1}}{\mu_{\infty}}\left(\frac{1}{\delta_{\mathrm{b}} \frac{\mathrm{d} v}{\mathrm{~d} \eta}} \tanh \left(\beta \delta_{\mathrm{b}} \frac{\mathrm{d} v}{\mathrm{~d} \eta}\right)+1\right)$

où

$$
\delta_{\mathrm{b}}=\frac{\mu_{1} V_{\mathrm{M}}}{\tau_{\mathrm{b}} R_{0}}
$$

- modèle de référence :

$$
\eta=-\frac{1}{4} \frac{\mathrm{d} v}{\mathrm{~d} \eta} \frac{\left(1-\frac{C}{2} k_{\infty}\right)^{2}}{\left[1-\frac{C}{2} \frac{k_{0}+k_{\alpha} \cdot\left(\delta_{\mathrm{q}}\left|\frac{\mathrm{d} v}{\mathrm{~d} \eta}\right|\right)^{1 / 2}}{1+\left(\delta_{\mathrm{q}}\left|\frac{\mathrm{d} v}{\mathrm{~d} \eta}\right|\right)^{1 / 2}}\right]^{2}}
$$


où

$$
\delta_{\mathrm{q}}=\frac{V_{\mathrm{M}}}{\dot{\gamma}_{\mathrm{c}} R_{0}} .
$$

A partir de ces relations il est alors très aisé de déterminer numériquement $\frac{\mathrm{d} v}{\mathrm{~d} \eta}(\eta)$, puis par une simple quadrature $v(\eta)$ :

$$
v(\eta)=\int_{1}^{\eta} \frac{\mathrm{d} v}{\mathrm{~d} \eta}\left(\eta^{\prime}\right) \mathrm{d} \eta^{\prime} .
$$

\subsection{MISE EN ÉVIDENCE DE L'EFFET NON NEWTONIEN} EN DIFFÉRENTS SITES DU SYSTÈME CIRCULATOIRE. En toute rigueur l'écoulement du sang ne peut être assimilé à un écoulement quasi stationnaire que dans le système veineux. Cependant, à titre de comparaison, on s'attachera, sans préjuger des résultats qui seront obtenus $(\S 4)$ en régime pulsatile, à présenter les résultats relatifs à la composante continue de l'écoulement pulsatile du système artériel.

Trois sites du système circulatoire humain sont ainsi présentés : l'aorte ascendante, les artérioles, la veine cave. En prenant des ordres de grandeurs de rayon et de vitesse moyenne admis habituellement dans la littérature [9], il est alors possible de comparer (Fig. 5) les profils de vitesse normalisés obtenus en chacun de ces sites, au profil de vitesse qui serait obtenu pour un fluide newtonien de viscosité $\mu_{\alpha}$, soumis au même gradient de pression.

La figure 5 appelle les commentaires suivants :

C'est dans les gros vaisseaux (veines, artères) que les effets non newtoniens sont les plus marqués; ce

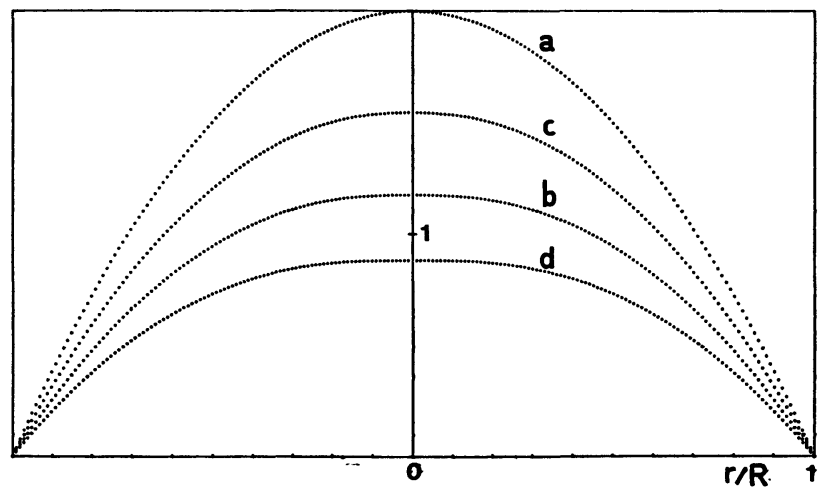

Fig. 5. - Comparaison des profils de vitesse normalisés avec le profil de vitesse $a$ ) obtenu pour un fluide newtonien de viscosité dynamique $\mu_{\infty} ; b$ ) Aorte ascendante chez l'homme, $V_{\text {Moy }}=$ $\left.2,5 \times 10^{-1} \mathrm{~m} / \mathrm{s}, R=1,25 \times 10^{-2} \mathrm{~m} ; c\right)$ Artériole : $V_{\text {Moy }}=10^{-2} \mathrm{~m} / \mathrm{s}$, $\left.R=10^{-4} \mathrm{~m} ; d\right)$ Veine cave inférieure, $V_{\text {Moy }}=1,2 \times 10^{-1} \mathrm{~m} / \mathrm{s}$, $R=1,75 \times 10^{-2} \mathrm{~m}$.

[Normalized velocity profiles of non newtonian fluids (b, c, d) in comparison with newtonian velocity profile $\left(a\right.$ : viscosity $\left.=\mu_{\infty}\right)$. b) Human ascending aorta (mean velocity $=2.5 \times 10^{-1} \mathrm{~m} / \mathrm{s}$, $R=1.25 \times 10^{-2} \mathrm{~m}$ ) $; c$ ) Arterioles (mean velocity $10^{-2} \mathrm{~m} / \mathrm{s}$, $\left.R=10^{-4} \mathrm{~m}\right) ; d$ ) Abdominal veina cava (mean velocity $\left.1.2 \times 10^{-1} \mathrm{~m} / \mathrm{s}, R=1.75 \times 10^{-2} \mathrm{~m}\right)$. Normalizing factor is the mean velocity of the newtonian profile (a).] résultat n'est pas paradoxal puisque c'est également dans les gros vaisseaux que les vitesses de cisaillement sont les plus faibles, et que le comportement non newtonien du sang se manifeste essentiellement à faible $\dot{\gamma}$.

Ces effets se traduisent par une augmentation de la viscosité apparente du fluide (diminution du débit à gradient de pression donné) et par un aplatissement du profil des vitesses dans la zone centrale. Cependant, il convient de remarquer que dans les sites artériels proches du cœur, le sang a été soumis à un brassage important. Ce brassage modifie l'état d'agrégation des particules (hématies), et change de manière sensible le comportement rhéologique du fluide, le retour à l'état d'agrégation à vitesse de cisaillement nulle s'effectuant en un temps de l'ordre de quelques secondes [8]. Compte tenu de la vitesse moyenne de l'écoulement, on conçoit aisément que les grosses artères sont concernées par ce phénomène. En faisant l'hypothèse que dans ces sites artériels, le sang est totalement désagrégé, on obtient alors (Fig. 6) un profil de vitesse où les effets non newtoniens sont sensiblement moins marqués.

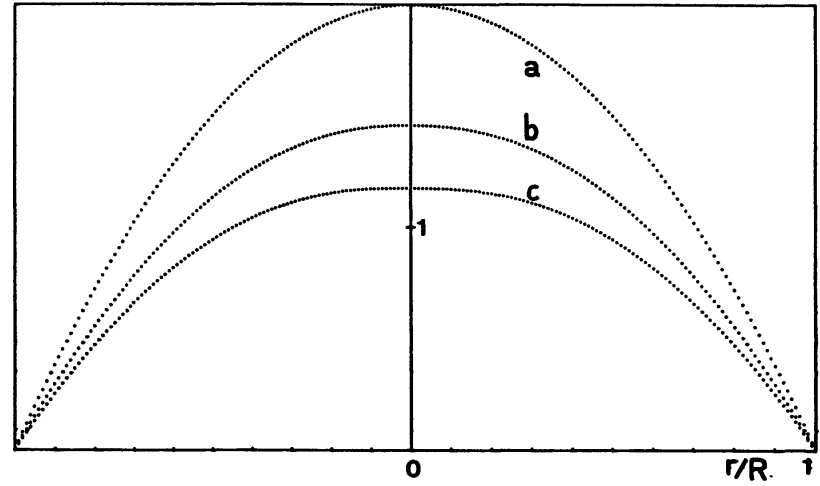

Fig. 6. - Comparaison des profils de vitesse normalisés obtenus pour du sang non désagrégé $(b)$ ou totalement désagrégé $(c)$ avec le profil de vitesse $(a)$ obtenu pour un fluide newtonien de viscosité dynamique $\mu_{\infty}$, dans le cas de l'aorte ascendante chez l'homme $\left(V_{\text {Moy }}=2,5 \times 10^{-1} \mathrm{~m} / \mathrm{s}, R=1,25 \times 10^{-2} \mathrm{~m}\right)$.

[Comparison between different normalised velocity profils (human ascending aorta, mean velocity $=2.5 \times 10^{-1} \mathrm{~m} / \mathrm{s}, R=1.25 \times 10^{-2} \mathrm{~m}$ ). a) Newtonian fluid (viscosity $=\mu_{\infty}$ ); $b$ ) Completely desaggregated human blood; c) Aggregated human blood.]

3.3 Couche Plasmatique. - On sait que la viscosité apparente des milieux dispersés est fonction de la concentration des particules en suspension. Or, au voisinage des parois, du fait même de la taille finie de ces particules, la concentration ne peut rester la même que dans le cœur de l'écoulement. D'autre part des effets de répulsion électrostatique tendent dans certaines circonstances à repousser les particules de la paroi. Enfin, les particules mises en rotation et placées au voisinage de la paroi dans un champ de cisaillement sont soumises à des forces hydrodynamiques qui les font migrer vers le centre de l'écoulement. 
Cette couche, pauvre en particules, appelée, dans le cas où la suspension considérée est du sang, couche plasmatique, a une épaisseur dont l'ordre de grandeur est de quelques $\mu \mathrm{M}$. Dans les vaisseaux de très faible diamètre, l'épaisseur de cette couche ne peut être négligée devant le rayon, elle joue en effet un rôle de lubrification, la viscosité dans la couche plasmatique étant sensiblement plus petite que dans le cœur de l'écoulement. Ainsi dans le cas de l'artériole qui était présenté (Fig. 5) il convient de prendre cet effet en compte, et l'on peut voir (Fig. 7) la modification importante apportée au profil des vitesses par la présence de cette couche.

Par contre, dans la grande circulation, son épaisseur devient petite devant le rayon du vaisseau, et il est habituel de la négliger.

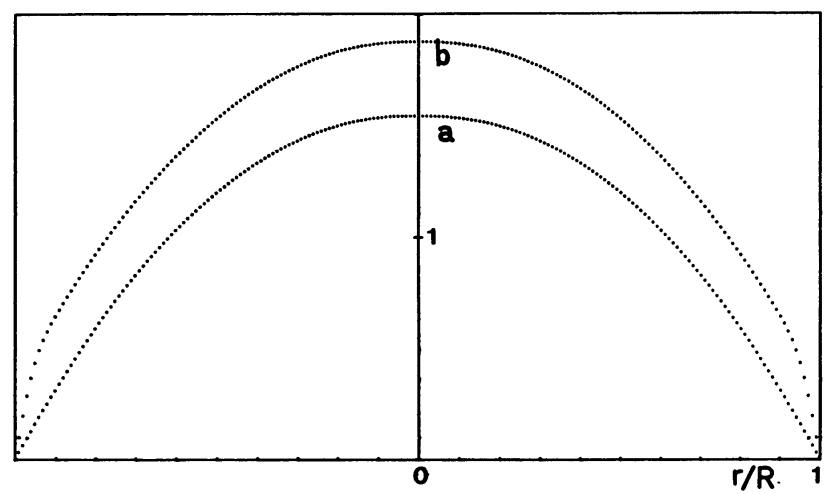

Fig. 7. - Modification du profil des vitesses résultant de la prise en compte de la couche plasmatique. Les profils de vitesse correspondent aux ordres de grandeur rencontrés dans des artérioles chez l'homme. Ces profils normalisés sont calculés pour le même gradient de pression sans tenir compte de la couche plasmatique $(a)$. La vitesse moyenne calculée vaut alors $10^{-2} \mathrm{~m} / \mathrm{s}$, ou bien en en tenant compte $\left(V_{\text {Moy }}=1,4 \times 10^{-2} \mathrm{~m} / \mathrm{s}\right)$.

[Modifications in normalised velocity profile, when taking into account marginal (plasma) layer (arteriols). Such velocity profiles have been calculated using the same pressure gradient with $(a)$ or without $(b)$ plasma layer $\left(R=10^{-4} \mathrm{~m}\right)$. The calculated mean velocities are $1.4 \times 10^{-2} \mathrm{~m} / \mathrm{s}(a)$ or $10^{-2} \mathrm{~m} / \mathrm{s}(b)\left(\nabla p=3 \times 10^{4} \mathrm{~Pa} / \mathrm{m}\right)$.]

\subsection{CHOIX DU MODÈLE RHÉOLOGIQUe EN ÉCOULE-} MENT PULSATILE. - Dans le cas où l'écoulement est dépendant du temps, il convient de discuter dans quelle mesure le caractère viscoélastique et thixotrope du fluide doit être pris en compte. On admettra, dans ce travail, que le caractère viscoélastique du sang qui est relié, entre autres, à la déformabilité des particules, peut être négligé. Il n'est pas possible, par contre, de ne pas tenir compte du caractère thixotrope du fluide qui est relié à l'état d'agrégation des particules. Cependant, compte tenu de l'ordre de grandeur du temps caractéristique de formation des agrégats, qui est de l'ordre d'une dizaine de secondes, on fera l'hypothèse qu'en un site donné du système circulatoire, cet état d'agrégation est indépendant du temps. En particulier, on peut supposer que, dans la grande

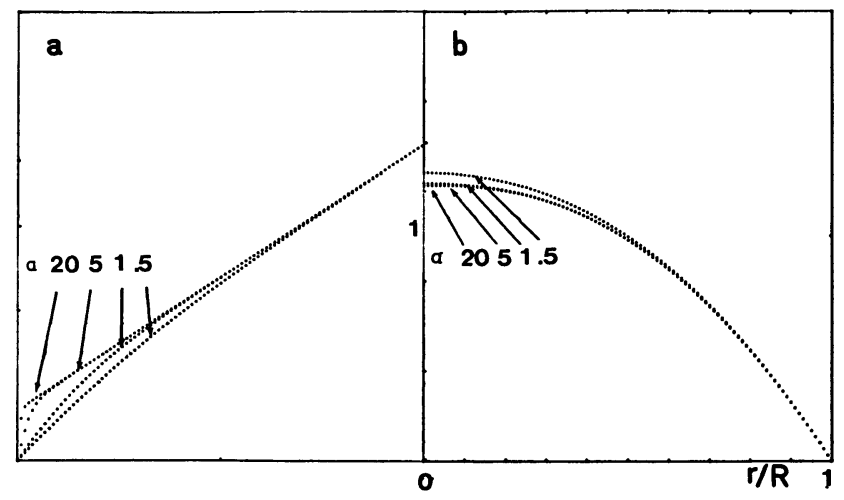

Fig. 8. - Influence de la viscosité apparente à $\dot{\gamma}=0$ sur la détermination des profils de vitesse. On a représenté $(8 a)$ les différentes relation $\tau(\dot{\gamma})$ obtenues (modèle de Casson continu) pour différentes valeurs du paramètre $\alpha(=20,5,1,0,5)$ c'est-à-dire pour différentes valeurs de la viscosité apparente à $\dot{\gamma}=0$. Parallèlement on montre $(8 b)$ les différents profils de vitesse obtenus pour chacune de ces valeurs. On s'est placé dans le cas où la vitesse moyenne de l'écoulement est de $0,12 \mathrm{~m} / \mathrm{s}$ et le rayon du tuyau de $6,7 \times 10^{-3} \mathrm{~m}$.

[Influence of zero shear apparent viscosity upon velocity profile determination. a) Casson diagram $\left(\tau^{1 / 2}\right.$ versus $\left.\dot{\gamma}^{1 / 2}\right)$ of continuous Casson model with different values of $\alpha$ i.e. different zero shear apparent viscosities. $b$ ) Corresponding normalised velocity profiles $\left.\left(\nabla p=110 \mathrm{~Pa} / \mathrm{m}, R=6.7 \times 10^{-3} \mathrm{~m}\right).\right]$

circulation artérielle, le sang reste peu agrégé, compte tenu du faible temps de transit entre le cour et le site d'étude (inférieur à 1 ou $2 \mathrm{~s}$ ), après le brassage important subi dans le ventricule gauche. Dans ce cas, les trois modèles rhéologiques présentés $(\$ 2)$ donnent des résultats comparables.

Cependant, pour des écoulements dépendant du temps, la détermination numérique des profils de vitesse pose des problèmes de convergence et de stabilité (cf. [3]) : la vitesse du calcul numérique doit en particulier être supérieure à la vitesse de diffusion de la vorticité; de telles considérations imposent que la condition :

$$
\frac{\mathrm{d} \eta^{2}}{\mathrm{~d} \theta}>40 \frac{v_{\mathrm{m}} T}{R^{2}}
$$

soit respectée. Dans cette relation $\mathrm{d} \eta$ représente le pas de calcul en espace, $\mathrm{d} \theta$ le pas de calcul en temps; $T$ la période de l'écoulement, $R$ le rayon du tuyau et $v_{\mathrm{m}}$ la viscosité cinématique maximale existant dans l'écoulement. Si on veut conserver un pas en espace suffisamment petit, on voit que le pas en temps est inversement proportionnel à la viscosité $v_{\mathrm{m}}$. Or dans le modèle de référence la viscosité apparente à vitesse de cisaillement nulle s'écrit :

$$
\mu_{0}=\frac{\mu_{\mathrm{p}}}{\left(1-\frac{C k_{0}}{2}\right)^{2}} .
$$

Avec les données numériques obtenues pour un sang non agrégé on obtient pour $\mu_{0}$ une viscosité de 
l'ordre de $15 \times 10^{-3}$ MKS qui impose des temps de calcul prohibitifs.

Or, en ce qui concerne la détermination des profils de vitesse pour des écoulements pour lesquels le domaine de variation des vitesses de cisaillement est assez étendu (vitesse de cisaillement maximale grande devant la vitesse de cisaillement $\dot{\gamma}_{\mathrm{c}}$ du modèle de référence), la valeur de la viscosité apparente à $\dot{\gamma}=0$ n'influe pas sensiblement sur les résultats. Ceci est illustré pour un écoulement continu (Fig. 8) où l'on a représenté pour un écoulement dont la vitesse moyenne est de $0,12 \mathrm{~m} / \mathrm{s}$ dans un tuyau de $6,7 \times 10^{-3} \mathrm{~m}$ de rayon, l'influence de la viscosité apparente à vitesse de cisaillement nulle, sur la détermination des profils de vitesse. Le modèle rhéologique utilisé est le modèle de Casson continu, qui permet, tout comme le modèle de Bingham continu, de déterminer simplement cette viscosité $\mu_{0}$ à l'aide du paramètre $\alpha$ ou $\beta$. On voit ainsi que $\mu_{0}$ peut varier de $+\infty$ (ce qui redonne le modèle de Casson) à $4 \mu_{\infty}$ sans modification sensible $\mathrm{du}$ profil des vitesses. On admettra par la suite la validité de ce résultat pour un écoulement pulsatile. Les mêmes conclusions peuvent être tirées pour le modèle de Bingham continu et c'est ce modèle, plus simple d'emploi, qui sera utilisé par la suite.

4. Ecoulements pulsatiles en tuyau souple. -4.1 PRISE. EN COMPTE DU CARACTÈRE NON NEWTONIEN DU FLUIDE. - Le caractère pulsatile de l'écoulement sanguin modifie profondément l'allure des profils de vitesse. Dans les grands vaisseaux et pour un paramètre de fréquence $\alpha_{\mathrm{f}}$ de l'ord re de $10\left(\alpha_{\mathrm{f}}^{2}=2 \pi R^{2} /(T v)\right.$ où $v$ est la viscosité cinématique du fluide), ce profil est très aplati dans la zone axiale, de telle sorte que, dans cette zone, l'écoulement s'effectue à de très faibles vitesses de cisaillement. La viscosité apparente y est donc élevée, et, de ce fait, les effets non newtoniens ont tendance à se manifester davantage dans cette zone où l'écoulement est dominé par des effets d'inertie (et à y renforcer l'aplatissement du profil des vitesses) que dans la couche limite oscillante dominée par les effets visqueux, mais où les vitesses de cisaillement sont importantes. On fera par la suite l'hypothèse que l'état d'agrégation du sang ne varie pas sensiblement en un site donné du système artériel. L'écoulement sera supposé pleinement développé et l'on cherchera à montrer que l'on ne peut négliger le caractère non newtonien du fluide dans certaines situations.

Ce problème est déjà ancien, puisqu'en 1959 [9], Taylor avait montré que, dans les grands vaisseaux les effets non newtoniens du fluide ne pouvaient modifier l'écoulement dans un tuyau rigide représentant les grosses artères que de $2 \%$ au maximum.

Cependant, il mérite d'être abordé quand le caractère viscoélastique non linéaire de la paroi est également pris en compte.

4.2 Formulation mathématiQue. - Or, on peut montrer [3] que dans l'étude des écoulements instationnaires pleinement développés, en tuyau souple, les équations gouvernant l'hydrodynamique du système se réduisaient à l'équation de continuité et à la composante axiale de l'équation de Navier-Stokes.

Compte tenu de la symétrie axiale, elles se réduisent à :

$$
\begin{aligned}
\rho\left(\frac{\partial w}{\partial t}+u \frac{\partial w}{\partial r}+w \frac{\partial w}{\partial z}\right) & =-\frac{\partial p}{\partial z}- \\
& -\left(\frac{1}{r} \frac{\partial}{\partial r}\left(r \tau_{r z}\right)+\frac{\partial \tau_{z z}}{\partial z}\right)=0 \\
\operatorname{div}(\mathbf{v}) & =0
\end{aligned}
$$

Dans cette expression $\vec{t}$ représente le tenseur des contraintes visqueuses. Or, de même que dans le cas où le fluide est newtonien, on peut écrire :

$$
\tau i j=-\mu\left(\frac{\partial v i}{\partial x j}+\frac{\partial v j}{\partial x i}\right)
$$

où $\mu$ n'est fonction que de la température et de la pression, de même dans le cas où le fluide est non newtonien, on posera :

$$
\vec{\tau}=-\mu\left\|\frac{\partial v i}{\partial x j}+\frac{\partial v j}{\partial x i}\right\|=-\mu \vec{\Delta}
$$

où $\mu$ est une fonction scalaire de $\vec{\Delta}$, donc de ses invariants $I_{1}, I_{2}, I_{3}([12])$ :

$$
\begin{aligned}
& I_{1}=\Sigma i \Delta i i \\
& I_{2}=\Sigma i \Sigma j \Delta i j \Delta j i \\
& I_{3}=\Sigma_{i} \Sigma_{j} \Sigma_{k} \varepsilon_{i j k} \Delta_{1 i} \Delta_{2 i} \Delta_{3 k} .
\end{aligned}
$$

Or, le premier invariant se réduit à $: I_{1}=2 \nabla \cdot \mathbf{v}=0$ puisque le fluide est supposé incompressible.

Dans un problème à symétrie axiale, le troisième invariant se réduit à :

$$
I_{3}=\frac{2 u}{r}\left(4 \frac{\partial u}{\partial r} \frac{\mathrm{d} w}{\partial z}-\left(\frac{\partial w}{\partial r}+\frac{\partial u}{\partial z}\right)^{2}\right) .
$$

Comme la vitesse radiale reste toujours très petite devant la vitesse longitudinale $w$, et que les dérivées selon la direction longitudinale sont très petites devant les dérivées selon la direction radiale, on peut écrire :

$$
\left.\begin{array}{c}
u \ll w \\
\frac{\partial}{\partial z} \ll \frac{\partial}{\partial r}
\end{array}\right\}
$$

l'expression du second invariant s'écrivant :

$$
I_{2}=4\left(\left(\frac{\partial u}{\partial r}\right)^{2}+\left(\frac{u}{r}\right)^{2}+\left(\frac{\partial w}{\partial r}\right)^{2}\right)+2\left(\frac{\partial u}{\partial z}+\frac{\partial w}{\partial r}\right)^{2}
$$


elle se réduit de la même manière à :

$$
I_{2}=2\left(\frac{\partial w}{\partial r}\right)^{2}=\vec{\Delta}: \vec{\Delta}
$$

$I_{1}$ étant nul $\mu$ n'est fonction que de $I_{2}$ et de $I_{3}$ :

$$
\mu=\mu\left(I_{2}, I_{3}\right) .
$$

Cependant dans le cas où le rayon du tube est constant, $I_{3}$ est nul et $\mu$ n'est fonction que de $I_{2}$. On supposera ici, comme il est communément admis, que $\mu$ reste indépendant de $I_{3}$ quand les variations de rayon sont faibles devant le rayon moyen du tube. On admettra donc, en définitive, que $\mu$ n'est fonction que de $I_{2}$ donc de $\partial w / \hat{\lambda}$.

4.3 EXPRESSION DE LA CONTRAINTE DE CISAILleMENT. - On a vu que l'on pouvait retenir, pour décrire de manière satisfaisante le comportement rhéologique du fluide, la relation :

$$
\tau=\left(\frac{\tau b}{\dot{\gamma}} \tanh \left(\beta \frac{\mu_{1} \dot{\gamma}}{\tau b}\right)+\mu_{1}\right) \dot{\gamma}=\mu \dot{\gamma} .
$$

Pour transposer ces relations à des relations tensorielles, compte tenu de la dépendance de $\mu$ en fonction du second invariant on écrira ([10]) :

$$
\begin{aligned}
\vec{\tau} & =-\mu \vec{\Delta} \\
& =-\left[\frac{\tau b}{\left(\frac{1}{2} \vec{\Delta}: \vec{\Delta}\right)^{1 / 2}} \tanh \left[\beta \frac{\mu_{1}}{\tau b}\left(\frac{1}{2} \vec{\Delta}: \vec{\Delta}\right)^{1 / 2}\right] \vec{\Delta}\right]
\end{aligned}
$$

Il est alors possible d'exprimer $\tau_{r z}$ et $\tau_{z z}$ qui interviennent dans l'écriture de l'équation de NavierStokes (10) :

$$
\begin{aligned}
& \tau_{r z}=-\mu\left(\frac{\partial w}{\partial r}+\frac{\partial u}{\partial z}\right) \simeq-\mu \frac{\partial w}{\partial r} \\
& \tau_{z z}=-\mu 2\left(\frac{\partial w}{\partial z}\right) \ll \tau_{r z}
\end{aligned}
$$

dans ces conditions de terme visqueux intervenant dans (10) s'écrit :

$$
\begin{aligned}
-\frac{1}{r} \frac{\partial}{\partial r}\left(r \tau_{r z}\right)-\frac{\hat{\zeta}}{\partial z}\left(\tau_{z z}\right) & =+\frac{\mu}{r} \frac{\partial w}{\partial r}+\frac{\partial}{\partial r}\left(\mu \frac{\partial w}{\partial r}\right) \\
& =+\frac{1}{r} \frac{\partial}{\partial r}\left(r \mu \frac{\partial w}{\partial r}\right)
\end{aligned}
$$

en définitive l'écriture de la composante longitudinale de l'équation de Navier-Stokes se réduira à :

$$
\begin{aligned}
\rho\left(\frac{\partial w}{\partial t}\right. & \left.+u \frac{\partial w}{\partial r}+w \frac{\partial w}{\partial z}\right)=-\frac{\partial p}{\partial z}+\frac{1}{r} \frac{\partial}{\partial r} \times \\
\times & {\left[r\left(\frac{\tau b}{\partial w / \partial r} \tanh \left(\frac{\beta \mu_{1}}{\tau b} \frac{\partial w}{\partial r}\right)+\mu_{1}\right) \frac{\partial w}{\partial r}\right] }
\end{aligned}
$$

soit :

$$
\begin{aligned}
& \rho\left(\frac{\partial w}{\partial t}+u \frac{\partial w}{\partial r}+w \frac{\partial w}{\partial z}\right)=-\frac{\partial p}{\partial z}+\frac{1}{r} \frac{\partial}{\partial r} \times \\
& \quad \times\left(r \mu_{\infty} \frac{\partial w}{\partial r}\right)+\frac{1}{r} \frac{\partial}{\partial r} \\
& \quad \times\left[r\left(\frac{\tau b}{\partial w / \partial r} \tanh \left(\beta \frac{\mu_{1}}{\tau b} \frac{\partial w}{\partial r}\right)+\left(\mu_{1}-\mu_{\infty}\right)\right) \frac{\partial w}{\partial r}\right]
\end{aligned}
$$

qui fait apparaître un terme correctif par rapport à l'expression obtenue dans le cas où le fluide est newtonien.

4.4 ECRITURE DU SYSTÈME D'ÉQUATION, ET PRINCIPE DE LA RÉSOLUTION NUMÉRIQUE. - Il convient d'adjoindre à l'équation (16), l'équation de continuité et les conditions aux limites. En faisant l'hypothèse que les déplacements longitudinaux de la paroi restent négligeables, et en écrivant $R$ comme une fonction de $z$ et de $t$, on peut écrire les conditions aux limites sous la forme :

$$
\begin{aligned}
& w(r=R, z, t)=0 \\
& u(r=R, z, t)=\frac{\partial R}{\partial t} .
\end{aligned}
$$

Afin de pouvoir effectuer commodément un maillage en espace et en temps pour la résolution numérique, il est utile d'effectuer le changement de variable suivant :

$$
\begin{aligned}
\eta & =\frac{r}{R(z, t)} \\
t^{\prime} & =t \\
z^{\prime} & =z .
\end{aligned}
$$

En utilisant ces variables le système d'équation se reécrit :

$$
\begin{aligned}
\rho\left(\frac{\partial w}{\partial t^{\prime}}+w \frac{\partial w}{\partial z^{\prime}}+\frac{u}{R} \frac{\partial w}{\partial \eta}-\frac{\eta}{R} \frac{\partial w}{\partial \eta}\left(\frac{\partial R}{\partial t^{\prime}}+w \frac{\partial R}{\partial z^{\prime}}\right)\right) & =-\frac{\partial p}{\partial z^{\prime}}+\frac{1}{R^{2}} \frac{1}{\eta} \frac{\partial}{\partial \eta}\left(\eta \mu_{\infty} \frac{\partial w}{\partial \eta}\right)+ \\
+ & \frac{1}{R^{2}} \frac{1}{\eta} \frac{\partial}{\partial \eta}\left(\eta \tau_{\mathrm{b}} \tanh \left(\frac{\beta \mu_{1}}{R \tau_{\mathrm{b}}} \frac{\partial w}{\partial \eta}\right)+\left(\mu_{1}-\mu_{\infty}\right) \eta \frac{\partial w}{\partial \eta}\right)
\end{aligned}
$$




$$
\begin{gathered}
\frac{1}{R}\left(\frac{\partial u}{\partial \eta}+\frac{u}{\eta}\right)+\frac{\partial w}{\partial z^{\prime}}-\frac{\eta}{R} \frac{\partial R}{\partial z^{\prime}} \frac{\partial w}{\partial \eta}=0 \\
u\left(\eta=1, z^{\prime}, t^{\prime}\right)=\frac{\partial R}{\partial t} \\
w\left(\eta=1, z^{\prime}, t^{\prime}\right)=0
\end{gathered}
$$

Dans ces expressions $\lambda w / \hat{\partial} z^{\prime}$ apparaît comme un terme correctif qu'il est intéressant d'approximer afin d'éliminer toute dépendance explicite en $z^{\prime}$ dans le système d'équation.

Pour l'écoulement d'un fluide newtonien dans un tuyau souple légèrement conique, il a été proposé [2] de donner une expression approchée de ce terme en écrivant :

$$
\frac{\partial w}{\partial z}=k(z, t)|w|
$$

soit en effectuant le changement de variable :

$$
\frac{\partial w}{\partial z^{\prime}}=\frac{\eta}{R} \frac{\partial w}{\partial \eta} \frac{\partial R}{\partial z^{\prime}}+k\left(z^{\prime}, t^{\prime}\right)|w|
$$

En utilisant les équations $(19 b)$ et $(19 c)$, il est possible d'expliciter $k\left(z^{\prime}, t^{\prime}\right)$, puis $u\left(\eta, z^{\prime} t\right)$. En reportant dans (19a) on obtient finalement :

$$
\begin{aligned}
\frac{\partial w}{\partial t^{\prime}}= & -\frac{1}{\rho} \frac{\partial p}{\partial z^{\prime}}+\frac{1}{R} \frac{\partial R}{\partial t^{\prime}}\left(\eta \frac{\partial w}{\partial \eta}-\frac{1}{\eta} \frac{\partial w}{\partial \eta} \frac{\operatorname{Sum}(\eta)}{\operatorname{Sum}(1)}+\frac{w|w|}{\operatorname{Sum}(1)}\right)+\frac{1}{R} \frac{\partial R}{\partial z^{\prime}} \times \\
& \times\left(\frac{2}{\eta} \frac{\partial w}{\partial \eta}\left(\operatorname{Su}(\eta)-\frac{\operatorname{Su}(1) \operatorname{Sum}(\eta)}{\operatorname{Sum}(1)}\right)+2 w|w| \frac{\operatorname{Su}(1)}{\operatorname{Sum}(1)}\right)+\frac{1}{\rho R^{2}} \frac{1}{\eta} \frac{\partial}{\partial \eta}\left(\eta \tau_{\mathrm{b}} \tanh \left(\frac{\beta \mu_{1}}{R \tau_{\mathrm{b}}} \cdot \frac{\partial w}{\partial \eta}\right)+\eta \mu_{1} \frac{\partial w}{\partial \eta}\right)
\end{aligned}
$$

où

$$
\begin{gathered}
S u(\eta)=\int_{0}^{\eta} \eta^{\prime} w \mathrm{~d} \eta^{\prime} \\
\operatorname{Sum}(\eta)=\int_{0}^{\eta} \eta^{\prime}|w| \mathrm{d} \eta^{\prime} .
\end{gathered}
$$

En supposant $\frac{\partial p}{\partial z}\left(z_{0}, t\right), \frac{\partial R}{\partial z}\left(z_{0}, t\right)$, et $R\left(z_{0}, t\right)$ connus, il est alors possible, connaissant $w\left(\eta, z^{\prime}, t^{\prime}\right)$ de déduire $w\left(\eta, z^{\prime}, t^{\prime}+\mathrm{d} t^{\prime}\right)$, en effectuant le calcul numérique aux différences finies.

On se donne au départ un profil des vitesses arbitraire, satisfaisant les conditions aux limites. Pour un gradient de pression périodique on obtient, au bout d'un certain nombre de cycles de calcul une solution stable qui a convergé vers la solution cherchée.

4.5 PRISE EN COMPTE DE LA RHÉOLOGIE PaRIÉTALE et RÉSultats du CAlCUl NUMÉrique. - Pour un matériau pariétal purement élastique $R$ peut également être considéré comme fonction de la pression $P$ et de $z$. Dans ces conditions on peut écrire :

$$
\frac{\partial R}{\partial z}=\frac{\partial R}{\partial \zeta}+\frac{i R}{\partial p} \frac{i p}{\partial \zeta}
$$

où $\partial R / \partial \zeta$ correspond à la prise en compte de la forme du tuyau au repos. $\partial R / \partial p$ est connu dès que les pro- priétés rhéologiques du matériau sont connues. On s'est placé ici, dans le cas simple où le matériau est élastique, linéaire (valeur du module d'Young $E=2 \times 10^{6} \mathrm{Nm}^{-2}$ comparable en ordre de grandeur à celui des parois artérielles). Pour un tuyau de section constante au repos, $\partial R / \partial z$ peut donc être relié directement aux propriétés rhéologiques du matériau pariétal.

En se donnant en outre la dépendance temporelle du gradient de pression (correspondant en ordre de grandeur à différentes données physiologiques ou physio-pathologiques) et la dépendance temporelle du rayon, il est alors possible de calculer l'évolution du profil des vitesses dans différents cas, et de comparer, en particulier, les résultats obtenus pour un fluide newtonien et pour un fluide non newtonien (de même viscosité $\mu_{\infty}$ pour les forts cisaillements). Cette comparaison appelle les commentaires suivants (Fig. 9).

Bien que les fluides soient soumis au même gradient de pression et s'écoulent dans des tuyaux identiques, 


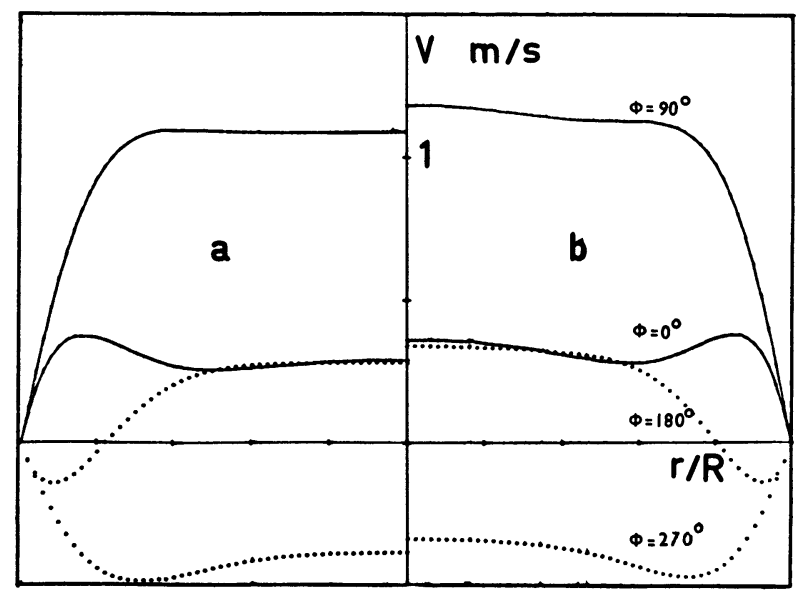

Fig. 9. - Comparaison des profils de vitesses obtenus, en tenant compte du caractère non newtonien du fluide $(a)$, et sans tenir compte de ce caractère $(b)$. On a pris un rayon de $R=6,67 \times 10^{-3} \mathrm{M}$, et une dépendance temporelle du gradient de pression donnée par $\Delta P(t)=(125,6+5385 \operatorname{Cos} \omega t) \mathrm{Pa} / \mathrm{M}$.

[Non newtonian pulsed velocity profiles $(a)$ in comparison with newtonian ones:

(b) $\left.\left(R=6.67 \times 10^{-3} \mathrm{~m}, \nabla P(t)=125+5800 \operatorname{Cos} \omega t(\mathrm{~Pa} / \mathrm{M})\right).\right]$

les débits associés au fluide non newtonien sont plus faibles que les débits associés au fluide newtonien. D'autre part les profils longitudinaux des vitesses sont plus aplatis dans la zone centrale. Les ordres de grandeur de rayon, de vitesse moyenne, de gradient de pression alternatif qui ont été choisis à titre d'illustration (artère chez l'homme) sont tels que le domaine de variation du gradient de vitesse $\left(0,300 \mathrm{~s}^{-1}\right)$ nécessite l'utilisation du modèle rhéologique étendu aux grandes vitesses de cisaillement présenté (\$2).

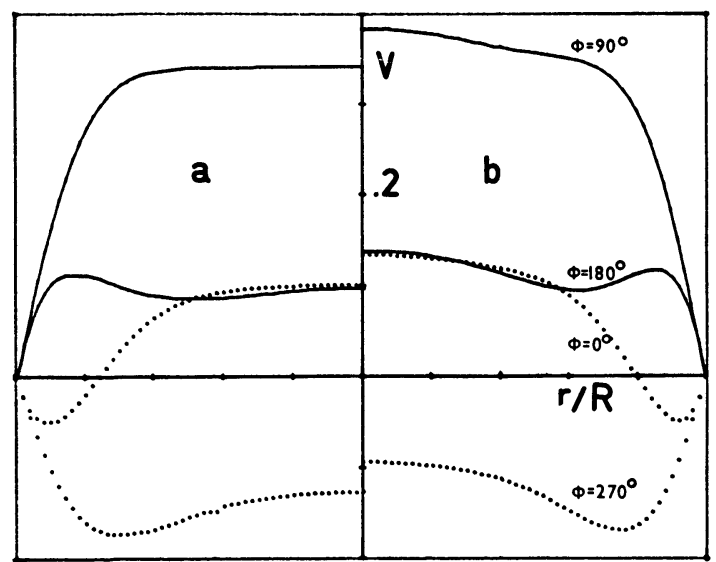

Fig. 10. - Comparaison des profils de vitesses obtenus pour un fluide non newtonien $(a)$ et newtonien $(b)$ pour l'écoulement d'un fluide (sang humain) dans un vaisseau de rayon $R=6,67 \times 10^{-2} \mathrm{M}$ sous l'action d'un gradient de pression sinusoïdal dont la dépendance temporelle s'écrit : $\Delta p=(41,8+1795 \operatorname{Cos} \omega t) \mathrm{Pa} / \mathrm{M}$.

[Non newtonian pulsed velocity profiles $(a)$ in comparison with newtonian ones;

(b) $\left(R=6.67 \times 10^{-3} \mathrm{~m}, \Delta P(t)=42+1790 \operatorname{Cos} \omega t(\mathrm{~Pa} / \mathrm{M})\right)$.]
On a également représenté (Fig. 10) les profils de vitesses obtenus pour un vaisseau de même rayon, mais pour lequel le gradient de pression a été divisé par un facteur 3 . On peut constater que dans ce cas les effets non newtoniens sont encore plus marqués et qu'ils ne peuvent être négligés. Cet exemple a été donné afin de montrer combien, dans des cas pathologiques où le débit est diminué de manière notable, la prise en compte du caractère non newtonien du fluide devient importante.

5. Conclusion. - En conclusion il doit être souligné que ce caractère non newtonien intervient dans la grande circulation, de manière marquée dans le système veineux, et de manière moins sensible dans la grande circulation artérielle. Cependant, il doit être pris en compte au même titre que l'élasticité de la paroi dès lors que le domaine de variation du gradient de vitesse reste limité à un domaine où la viscosité apparente s'écarte de manière sensible de la viscosité $\mu_{\infty}$ à gradient de vitesse infini.

Remarquons de plus qu'il est possible de traduire de manière satisfaisante l'écoulement du fluide non newtonien à l'aide d'une description où le fluide est considéré comme newtonien, mais où on lui attribue une viscosité effective $\mu_{\text {eff }}$ telle que dans les deux cas l'amplitude du débit alternatif est la même (Fig. 11). Cette viscosité $\mu_{\text {eff }}$ n'est alors qu'une extension dans

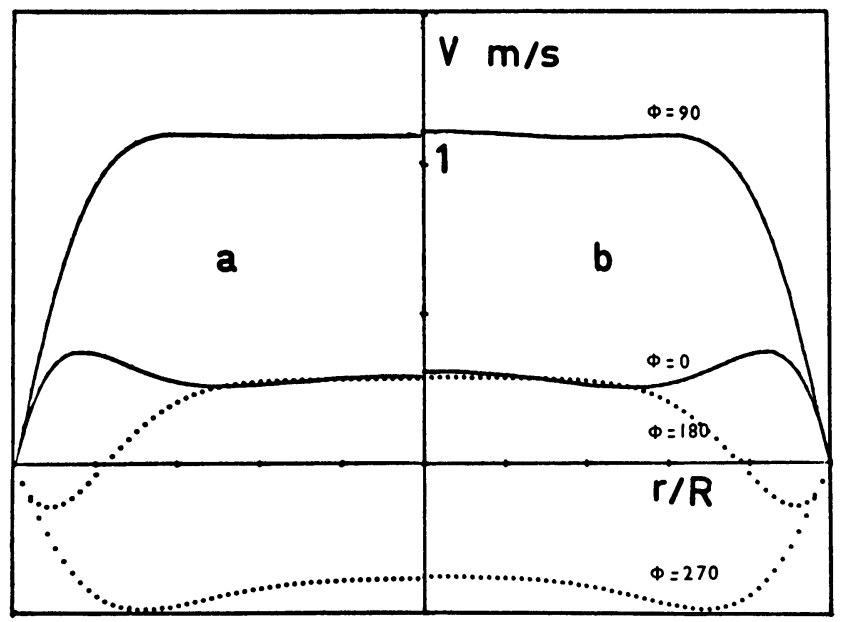

Fig. 11. - Comparaison des profils de vitesses obtenus pour un fluide non newtonien de viscosité $\mu_{\infty}=3,14 \times 10^{-3} \mathrm{MKS}$ et pour un fluide newtonien de viscosité $\mu_{\text {eff }}=3,3 \times 10^{-3}$ MKS. L'écoulement du fluide (sang humain) s'effectue dans un vaisseau de rayon $R=6,67 \times 10^{-1} \mathrm{M}$, sous l'action d'un gradient de pression sinusoïdal dont la dépendance temporelle s'écrit :

$$
\Delta p(125,6+5385 \operatorname{Cos} \omega t) \mathrm{Pa} / \mathbf{M} .
$$

[Pulsed velocity profile of non newtonian fluid

$$
\left(\mu_{\infty}=3.14 \times 10^{-3} \mathrm{MKS}\right)
$$

in comparison with pulsed velocity profile of newtonian fluid $\left(\mu=3.75 \times 10^{-3} \mathrm{MKS}\right)$

$$
\left.\left(R=6.67 \times 10^{-3} \mathrm{~m}, \nabla p=125+5800 \operatorname{Cos} \omega t(\mathrm{~Pa} / \mathrm{M})\right) .\right]
$$


le cas des tuyaux souples, de la viscosité apparente $\mu_{\mathrm{a}}$ définie pour des fluides non newtoniens en tuyau rigide.

Dans le cas ([3]) où l'on s'intéresse à la détermination indirecte des propriétés rhéologiques du fluide et de la paroi à partir de la mesure des profils de vitesse longitudinale, et où l'on suppose que le fluide est newtonien, c'est donc cette viscosité effective $\mu_{\text {eff }}$ que l'on déterminera, qui peut varier en fonction du site artériel où est effectuée la mesure.

Soulignons enfin que ces résultats reposent sur l'hypothèse simplificatrice permettant de négliger les effets viscoélastiques et thixotropes. Une telle hypothèse demanderait une analyse plus détaillée du rôle de ces effets dans les écoulements considérés. Cette analyse est en cours.

\section{Bibliographie}

[1] MCDonald, D. A., Blood flow in arteries (London-Edinburg, Edward Arnold) 1974

[2] Ling, S. C., AtabeK, H. B., A non linear analysis of pulsatile flow in arteries, J. Fluid Mech. 55 (1972) 493-511.

[3] Flaud, P., Influence des propriétés rhéologiques non linéaires sur la dynamique des écoulements dans un tuyau déformable. Thèse de Doctorat, Paris 1979.

[4] CoKelEt, G. R., The rheology of human blood. Biomechanics, its foundations and objectives (Fung, Perrone, Anliker Ed. Prentice Hall, New Jersey) 1972, 63-103.

[5] CASsON, N., A flow equation for pigment-oil suspensions of the printing ink type. Rheology of disperse systems (Pergamon Press, London) $1959,84$.

[6] Merril, E. D., Pelletier, G. A., Viscosity of human blood : transition from newtonian to non newtonian, J. Applied Physiol. 23 (1967) 178-182.
[7] Quemada, D., Rheology of concentrated disperse system. Rheol. Acta 16 (1977) 82-94; 17 (1978) 632-642; 17 (1978) 643-653.

[8] Mills, P., Adler, P., Quemada, D., Dufaux, J., Etude de l'agrégation d'une suspension sanguine par rétrodiffusion Laser. Journal des maladies cardiovasculaires 4 (1979) 91-94.

[9] TAYLOR, M. G., The influence of the anomalous viscosity of blood upon its oscillatory flow, Phys. Med. Biol. 3 (1959) 273-290

[10] Bird, R., SteWART, W., LightFoot, E., Transport phenomena (John Wiley Ed. New York) 1977. 